This is an electronic reprint of the original article. This reprint may differ from the original in pagination and typographic detail.

\author{
Author(s): Tuononen, Heikki; Chivers, Tristram; Armstrong, Andrea; Fedorchuk, Chantall; Boeré, \\ René
}

Title: $\quad$ Computational modeling of isotropic electron paramagnetic resonance spectra of doublet state main group radicals

Year: $\quad 2007$

Version:

Please cite the original version:

Tuononen, H., Chivers, T., Armstrong, A., Fedorchuk, C., \& Boeré, R. (2007).

Computational modeling of isotropic electron paramagnetic resonance spectra of doublet state main group radicals. Journal of Organometallic Chemistry, 692(13), 2705-2715. https://doi.org/10.1016/j.jorganchem.2006.12.019

All material supplied via JYX is protected by copyright and other intellectual property rights, and duplication or sale of all or part of any of the repository collections is not permitted, except that material may be duplicated by you for your research use or educational purposes in electronic or print form. You must obtain permission for any other use. Electronic or print copies may not be offered, whether for sale or otherwise to anyone who is not an authorised user. 


\title{
Computational modeling of isotropic electron paramagnetic resonance spectra of doublet state main group radicals ${ }^{\dagger}$
}

\author{
Heikki M. Tuononen ${ }^{\mathrm{a}, *}$, Tristram Chivers ${ }^{\mathrm{b}}$, Andrea Armstrong ${ }^{\mathrm{b}}$, Chantall Fedorchuk ${ }^{\mathrm{b}}$ \\ and René T. Boeré ${ }^{\mathrm{c}}$ \\ ${ }^{a}$ Department of Chemistry, University of Jyväskylä, Jyväskylä, Finland FI-40014. \\ ${ }^{\mathrm{b}}$ Department of Chemistry, University of Calgary, Calgary, Alberta, Canada T2N 1N4. \\ ${ }^{c}$ Department of Chemistry and Biochemistry, University of Lethbridge, Lethbridge, \\ Alberta, Canada T1K 3M4.
}

* Corresponding author.

Tel.: +358-14-260-2618, Fax: +358-14-260-2501, E-mail: hetuonon@cc.jyu.fi.

$\uparrow^{\dagger}$ This article is based on the oral presentation presented by Dr. Heikki M. Tuononen (Abstract 143) at the $11^{\text {th }}$ International Symposium on Inorganic Ring Systems, Oulu, Finland, July 30 - August 4, 2006. 


\begin{abstract}
The combined use of theoretical and mathematical methods in the analysis of electron paramagnetic resonance data has greatly increased the ability to interpret even the most complex spectra reported for doublet state inorganic main group radicals. This personal account summarizes the theoretical basis of such an approach and provides an in-depth discussion of some recent illustrative examples of the utilization of this methodology in practical applications. The emphasis is on displaying the enormous potential embodied within the approach.
\end{abstract}

Keywords: EPR spectroscopy; Main group radicals; Spectral simulation; DFT calculations

\title{
1. Introduction
}

Isotropic (solution state) electron paramagnetic resonance (EPR) spectra of doublet state organic radicals are usually simple to interpret. There are two main arguments which promote the above statement: from the different nuclei typically present in organic systems, only hydrogen and nitrogen have spin-active isotopes with significant (approx. 100\%) natural abundances, and the values of nuclear spin for both ${ }^{1} \mathrm{H}$ and ${ }^{14} \mathrm{~N}$ nuclei are low, $I=1 / 2$ and 1 , respectively [1]. In the majority of cases, this leads to simple and easily detectable splitting patterns in the experimental spectrum [2]. Conversely, isotropic EPR spectra of inorganic main group radicals including other s- and p-block elements than hydrogen and nitrogen are often poorly resolved due to the hyperfine coupling ( $\mathrm{hfc}$ ) of the unpaired electron to magnetically active nuclei with large nuclear spin values $(I>1)$ and more than one naturally abundant spin-active isotope (see Figure 1) [1].

The complexity in the EPR spectra of main group radicals containing multiple spinactive nuclei generally renders it difficult to extract accurate values of hyperfine coupling (hfc) constants from the experimental spectrum. Since such data is used to gather information of the spin distribution within a paramagnetic molecule, this is quite problematic, as it can impede researchers from gaining a thorough understanding of a 
particular radical system. In addition, without any knowledge of the hfc constants, it is impossible to produce a simulation of the experimental spectrum which will most likely prevent the identification of the observed radical species. Thus, it is evident that accurate hyperfine parameters play a prominent role in the study of paramagnetic systems.
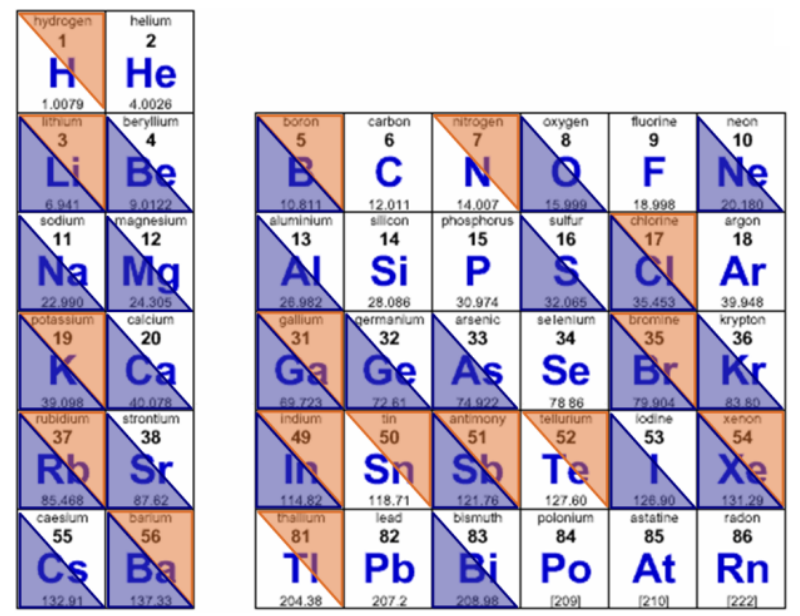

Fig 1. The 42 different s- and p-block elements. Lower triangle denotes an element for which nuclear spin is greater than one and upper triangle denotes an element with more than one spin-active isotope.

One possible and very often used method to overcome the above difficulties in spectral interpretation is to employ theoretical first principles methods to calculate the magnitudes of the hfcs and then compare these results to data extracted from the experimental spectrum [3]. In fact, there is a long history of using theoretical calculations in the interpretation of EPR spectra as perturbation molecular orbital and semi-empirical methods have been used as early as 50's and 60's [4]. However, an even more powerful approach is to employ the calculated hfc constants as initial estimates of the true spectral parameters and then use iterative least-squares fit based methods to automatically refine the simulation with respect to the experimental EPR spectrum. We have recently shown that this technique can be an extremely successful tool in the assignment and interpretation of complex EPR spectra reported for inorganic main group radicals [5]. This short personal account briefly reviews the theoretical basis of the approach and summarizes the results from its application to the analysis of some paramagnetic systems. 


\section{Theoretical and computational considerations}

\section{Theoretical methods for calculation of isotropic hyperfine coupling constants}

The $3 \times 3$ hyperfine coupling tensors $A(i)$ describe the interaction of the unpaired electron with the spin-active nuclei $i$ [6]. They can be separated into isotropic and anisotropic components of which only the former is discussed herein. A good (first-order) approximation of the isotropic hfc $A_{\text {iso }}(i)$ for nucleus $i$ is given by the Fermi contact interaction term

$$
A_{\mathrm{iso}}(i)=\frac{8 \pi}{3} g_{e} \beta_{e} g_{i} \beta_{n}\left\langle\Psi\left|\delta\left(r_{i}\right) S_{z}\right| \Psi\right\rangle=\frac{8 \pi}{3} g_{e} \beta_{e} g_{i} \beta_{n} \rho\left(r_{i}\right),
$$

where $\beta_{n}$ is the nuclear magneton, $g_{e}$ is the electronic $g$-factor, and $g_{i}$ and $\rho\left(r_{i}\right)$ are the nuclear $g$-factor and electron spin density at the nucleus, respectively, for nucleus $i$. Simply put, hfc constants are obtained by multiplying the electronic spin density evaluated exactly at each nucleus $i$ with the appropriate physical constants. As suggested by the form of the Fermi contact term, the connection between spin density and hfcs can also be used in the opposite order i.e. experimentally determined hfc constants provide a practical means for evaluation of nuclear spin densities.

From the form of the Fermi contact interaction term, it follows that the hfc constants are extremely difficult to calculate theoretically because of their high sensitivity to the quality of the wave function (spin density) at one point in space; the Dirac delta function $\delta\left(r_{i}\right)$ evaluates the wave function only at the nucleus thus making the property very local and unlikely to benefit from error cancellations. Although alternative formulations which use more global operators than the Dirac delta function in calculation of Fermi contact interaction have been introduced [7], none of them have yet found widespread use.

The delta function-based formulation also implies that the Gaussian-type (GTO) basis sets employed in the majority of molecular orbital methods are fundamentally flawed to be used in calculation of Fermi contact interactions [8]. However, it has been shown that, when augmented with tight s-functions, the standard Gaussian-type basis sets can indeed overcome the nuclear cusp problem [9]. An exhaustive number of calculations have also demonstrated that the EPR-III basis sets perform well in density functional 
theory (DFT) calculations [10], as do the more conventional TZVP [10,11], while the IGLO basis sets generally give good magnetic properties with a reasonable basis set size in $a b$ initio calculations [12]. It should also be pointed out that specifically tailored GTO basis sets for the calculation of hfc constants of main group radicals exist only for Period 1 and 2 nuclei (EPR-II and EPR-III) [10a]. In addition, the performance of the TZVP basis sets (and some of their variants) in DFT based calculations has been statistically analyzed only up to Period 3 nuclei silicon, phosphorus and sulfur [10c]; there exists only a limited number of main group radicals in which significant spin density is found on nuclei from fourth (or higher) Period and whose hfc constants are known experimentally with sufficient accuracy to facilitate comparison with calculated results [13].

For systems with heavier s- and p-block elements (Period 4 and beyond), relativistic effects need to be treated appropriately [14]. In principle, this can be done in both wave function and DFT based approaches. Relativistic effects can also be approximated in theoretical calculations by using pseudopotential basis sets for heavy elements. In this quasi-relativistic approach, the replacement of core electrons of the heavy element with an effective potential allows modeling of the effects of relativity only to the nearby lighter nuclei and actually prevents the determination of hfc constants to the heavy element itself as the inner s-electrons are not treated implicitly in the calculation.

In order to obtain accurate results, $a b$ initio calculations of hfc constants need to be conducted using correlated levels of theory [15]. On purely theoretical grounds, it is, however, not immediately obvious which of the many modern density functionals is most appropriate to use in DFT calculations of hfc constants [16]. The choice of a functional is therefore strongly influenced by its reported performance. In general, generalized gradient approximation (GGA) functionals depending also on the kinetic energy density (meta-GGA) lead usually to small or no improvements over the basic GGA approximation [17], and hybrid functionals tend to yield the best results for a wide variety of main group systems $[10,18]$. It should also be noted that reproducing trends in hfc constants as functions of structural parameters is a much easier task than matching the absolute values with experimental data. Such correlations are often achieved with almost any number of functionals [19]. 


\section{Mathematical methods for spectral simulation}

The accurate extraction of relevant physical parameters from experimental data comprises a major problem in all kinds of spectroscopic methods. The analysis of an experimental spectrum is generally a deceivingly straightforward procedure for simple molecular systems, but can become a very challenging problem when the system size is increased or the identity of even some of the atoms in a molecule is changed. As exemplified in the introduction, EPR spectroscopy of doublet state main group radicals is no exception to this rule: the presence of multiple low-spin or even a few high-spin ( $I>$ 1) nuclei can lead to extremely complex and, therefore, incompletely resolved hyperfine coupling patterns in which the majority of the information describing the paramagnetic species is lost under the broad lineshape. In such cases, simple trial-and-error based simulation attempts are clearly inadequate, since minor errors in the parameters can alter the appearance of a simulated spectrum significantly. Therefore, efficient spectral fitting algorithms augmented with an automated refinement of the parameters used in the simulation are prerequisites for successful simulation of complex EPR spectra [20].

All spectral fitting programs are based on the repeated utilization of three basic steps, simulation, comparison and refinement, which are iterated in an ordered fashion until either the maximum number of steps is reached or the procedure converges to a solution for which the calculated parameter indicating the goodness of the fit is lower than a pre-defined threshold. In the context of isotropic EPR spectra of doublet radicals, the above is nothing more complex than performing a nonlinear least-squares fit in a multidimensional parameter space in which the parameters are the number and identity of spin-active nuclei $i$, the isotropic hyperfine coupling constants $A_{\text {iso }}(i)$, the $g$-value of the radical, as well as both the spectral line-width and amplitude [21,22]. As this problem is very general in nature, several algorithms designed to perform such fitting have been described in the mathematical literature, e.g. simplex, Monte-Carlo, Hooke-Jeeves and Levenberg-Marquardt [23], and are readily available in several of the more advanced simulation software designed for the needs of EPR spectroscopists (e.g. XSophe, EasySpin and EPR-Winsim) [24]. We note here that the simplex algorithm is especially well suited to the least-squares analysis of highly complex EPR spectra [25] and has, therefore, also been used in the selected examples described in the next section. 
Despite the immense advantages that the use of iterative least-square based fitting algorithms offer, their use in spectral analysis also introduces some problems: it is in general impossible to build an automated simulation program which, in all possible cases, would lead to an unambiguous set of chemically relevant hfes and other spectral parameters that would reproduce the experimental spectrum with great precision. This arises in part from problems associated with the fitting algorithms themselves (failure of convergence or convergence to an undesired local minimum), but also from the limitations imposed by the quality of the experimental spectrum (poorly resolved lines due to spectral overlap or imperfect measuring conditions).

Even though the experimental EPR spectrum would be of perfect quality, there exists no such least-squares fit algorithm which would be able to converge to an unambiguous solution in a reasonable amount of time without good initial estimates of the individual parameters used in the iterative optimization. Whereas it is relatively straightforward to determine the proper linewidth, $g$-value and amplitude from the experimental spectrum, it is in most cases rather difficult to come up with reasonable estimates for the individual hyperfine coupling constants. After all, these are the key parameters that the iterative procedure is supposed to determine in the first place! For a long time there were only two practical methods available to produce the required estimates of hfcs: sophisticated guesses based on chemical knowledge of the system and the use of hfc constants determined via electron-nuclear double resonance (ENDOR) spectroscopy [26]. A third, much more modern, approach is to employ high-frequencyhigh-field EPR spectroscopy (W- and Q-bands). The advent of high-performance computers has, however, introduced another, more easily applicable, possibility to determine a suitable set of initial hfc constants by performing theoretical calculations for radical species in question. Statistical analyses of results have shown that the accuracy of the most common methods in predicting hfc constants is as good as $5-10 \%$ and $10-20 \%$ for Period 1 and 2 nuclei [10,15,16] respectively, which in the majority of cases facilitates smooth convergence of the iterative least-squares fit procedure to a global minimum. Despite the long-term general availability of efficient quantum chemical codes capable of performing hfc constant calculations, an approach that combines computational results with mathematical methods has not yet, however, found widespread usage in practical applications. 


\section{Illustrative examples}

In the following sections we present three examples of studies in which theoretical calculations have been used in combination with mathematical spectral optimization methods to aid in the analysis of some very complex EPR spectra reported for inorganic main group radicals.

All spectra were recorded using Bruker EMX113 and ESP300E spectrometers operating in the X-band. Spectral simulations were carried out with the XEMR [27] and WINEPR SimFonia programs [28]. Hyperfine coupling constant calculations were done at the PBE0 (non-relativistic) and PBEPBE (relativistic) levels of theory [29] using Gaussian 03 [30] and ADF 2005.01 [31] program packages, respectively.

\section{Spirocyclic group 13-boraamidinate radicals}

Treatment of the dimeric dilithiated boraamidinate $\left\{\mathrm{Li}\left[\mathrm{PhB}\left(\mathrm{N}^{t} \mathrm{Bu}\right)_{2}\right]\right\}_{2}$ with the metal halides $\mathrm{MCl}_{3}(\mathrm{M}=\mathrm{Al}, \mathrm{Ga}$, In) in a 1:1 molar ratio produces the spirocyclic anions $\left[\mathrm{PhB}\left(\mu-\mathrm{N}^{\mathrm{t} B u}\right)_{2} \mathrm{M}\left(\mu-\mathrm{N}^{\mathrm{t}} \mathrm{Bu}\right)_{2} \mathrm{BPh}\right]^{-}(\mathbf{1 b}, \mathrm{M}=\mathrm{Al} ; \mathbf{1 c}, \mathrm{M}=\mathrm{Ga} ; \mathbf{1 d}, \mathrm{M}=\mathrm{In})$ [32]. In the solid state, the anions chelate a solvated lithium cation via two nitrogen atoms; in solution, the lithium cation exchanges rapidly on the NMR timescale between coordination to different pairs of $\mathrm{N}^{t} \mathrm{Bu}$ nitrogen atoms. An analogous anionic boron-centered spirocycle $[\mathrm{PhB}(\mu-$ $\left.\left.\mathrm{N}^{\mathrm{t} B u}\right)_{2} \mathrm{~B}\left(\mu-\mathrm{N}^{\mathrm{t}} \mathrm{Bu}\right)_{2} \mathrm{BPh}\right]^{-}$(1a) was recently shown to be the minor product of a reaction between $\left\{\mathrm{Li}\left[\mathrm{PhB}\left(\mathrm{N}^{t} \mathrm{Bu}\right)_{2}\right]\right\}_{2}$ and boron trifluoride $\mathrm{BF}_{3}$ in a 1:2 molar ratio [5d].

An intriguing observation in the synthesis of systems $\mathbf{1}$ was the formation of intensely colored solutions when the reaction mixtures were briefly exposed to air [32]. The formation of radical species was confirmed by EPR spectroscopy and the paramagnetic nature of the solutions was thought to arise from the formation of an anion radical $\left\{\left[\mathrm{PhB}\left(\mathrm{N}^{t} \mathrm{Bu}\right)_{2}\right]\right\}^{-}$. As the resulting EPR spectra were found to be extremely complex, the oxidation reactions of compounds $\mathbf{1}$ were performed in a controlled manner using half an equivalent of iodine and the EPR spectra were re-determined. Although the new spectra showed more details in their fine structure, the overall shapes of the signals in the two sets of spectra remained identical. 


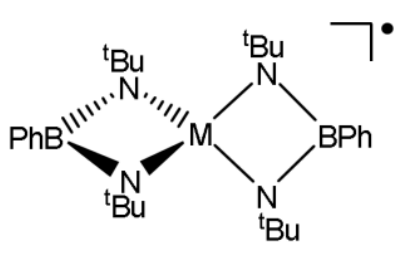

2

The iodine oxidation reactions of $\mathbf{1}$ should eliminate lithium iodide and yield the neutral radicals $\left[\mathrm{PhB}\left(\mu-\mathrm{N}^{\mathrm{t}} \mathrm{Bu}\right)_{2} \mathrm{M}\left(\mu-\mathrm{N}^{\mathrm{t}} \mathrm{Bu}\right)_{2} \mathrm{BPh}\right]^{\bullet}(\mathbf{2 a}, \mathrm{M}=\mathrm{B} ; \mathbf{2} \mathbf{b}, \mathrm{M}=\mathrm{Al} ; \mathbf{2} \mathbf{c}, \mathrm{M}=\mathrm{Ga}$; 2d, $\mathrm{M}=\mathrm{In}$ ) which are expected to retain the spirocyclic geometry of their diamagnetic precursors both in solution and in the solid state. To aid in the spectral analysis, DFT calculations were carried out for the model systems [33] $\left[\mathrm{PhB}(\mu-\mathrm{NMe})_{2} \mathrm{M}(\mu-\mathrm{NMe})_{2} \mathrm{BPh}\right]^{\cdot}$ to determine their electronic structures and spin densities $[5 \mathrm{c}, \mathrm{d}]$. The results indicated that the radicals indeed have stable $D_{2 \mathrm{~d}}$ symmetric structures with ${ }^{2} \mathrm{~A}_{2}$ ground states and display uniform spin delocalization throughout the two boraamidinate ligands. The analysis of the spin density distributions revealed that if the radicals $\mathbf{2}$ exist in spirocyclic geometries also in solution, their EPR spectra should exhibit hfcs to the central element $\mathrm{M}$ as well as to four and two equivalent ${ }^{14} \mathrm{~N}$ and ${ }^{10,11} \mathrm{~B}$ nuclei, respectively. Although hfcs to the spin-active elements in the boraamidinate ligands were predicted to be very similar among the four isostructural compounds (see below) which should simplify the spectra, an additional level of complexity is, however, introduced by the presence of different isotopes: according to the first-order hfc rules, the EPR spectra of these radicals actually consist of as many as $4752(\mathrm{~B}), 2592(\mathrm{Al}), 3456(\mathrm{Ga})$ and 8640 (In) individual lines. However, due to the low natural abundance of ${ }^{10} \mathrm{~B}(19.80 \%)$ and ${ }^{113}$ In nuclei $(4.28 \%)$, the experimental spectra are largely dominated by subspectra belonging to isotopomers which contain only the ${ }^{11} \mathrm{~B}$ and ${ }^{115} \mathrm{In}$ nuclei

The experimental EPR spectra of 2a-d are shown in Figure 2a [5c,d;34]. It is evident that the number of resolved lines in all four spectra is considerably less than predicted for narrow linewidths. In addition, only the spectrum of the indium species is dominated by a feature whose origin is immediately apparent: the decet of multiplets due to hfc to the ${ }^{115}$ In nuclei $(I=9 / 2)$. Hence, there is an extensive overlap of lines in each spectrum which suggests that simulations need to be carried out using iterative leastsquare fit methods employing calculated hfcs as initial estimates of the true couplings 
present in 2. The calculated hfcs as well as those used in the final simulations are collected to Table 1; the simulated EPR spectra are shown in Figure 2b [5c,d].

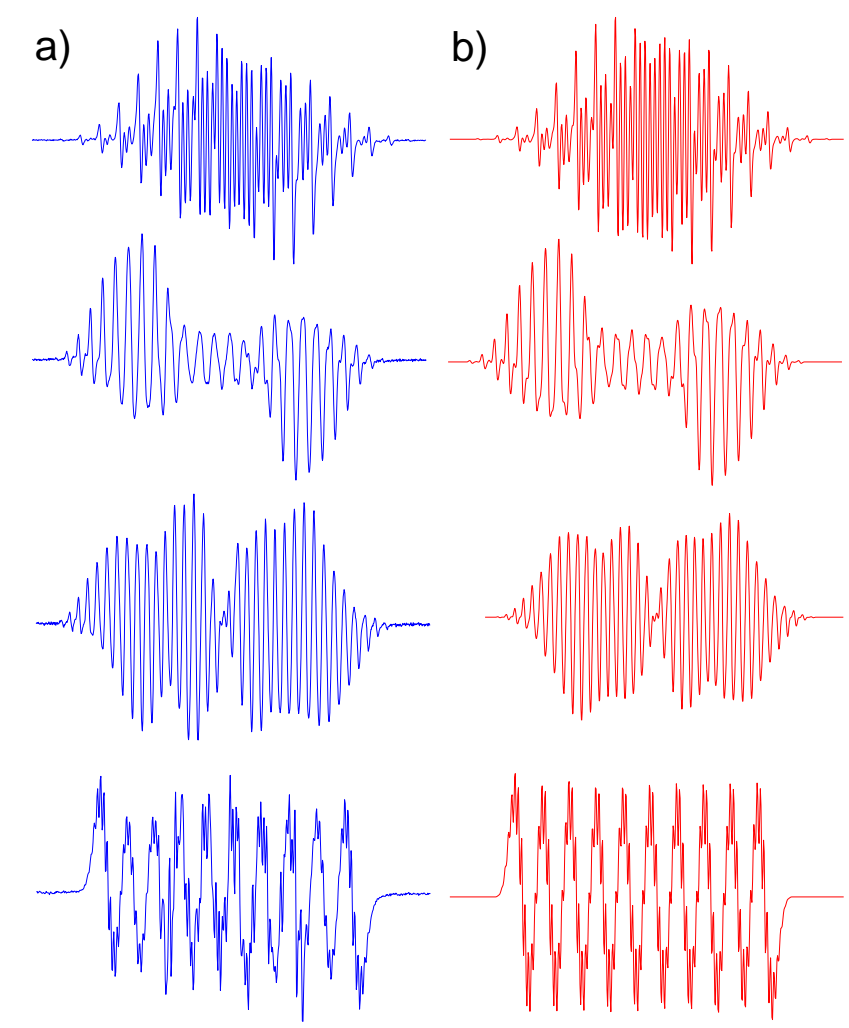

Fig. 2. Experimental (a) and simulated (b) EPR spectra of radicals 2a (top) - 2d (bottom) $[5 \mathrm{c}, \mathrm{d}]$.

A comparison of the simulated spectra with their experimental counterparts reveals excellent agreement. The calculated hfc constants are also reasonably close to the optimized values: the agreement is very good for the ${ }^{14} \mathrm{~N}$ and ${ }^{10,11} \mathrm{~B}$ nuclei, but less satisfactory for the heavier central metals aluminum and, especially, gallium. We note that this discrepancy arises most likely from the use of standard, energy-optimized, basis sets for these elements in theoretical calculations [5c,d]. Comparing the computational results with the experimental data provides conclusive evidence that the oxidation reactions give the expected radicals which have the proposed spirocyclic geometries in solution. Further evidence supporting this result came from subsequent X-ray analyses which were performed for crystals grown from concentrated diethyl ether solutions of aluminum and gallium compounds $\mathbf{2 b}$ and $\mathbf{2 c}$ [5c]: the structural determinations 
confirmed that the two complexes are indeed isostructural and exist in the delocalized spirocyclic geometries in the solid state.

\section{Table 1}

Experimental and calculated hyperfine coupling constants (in Gauss) of radicals $\mathbf{2}^{\mathrm{a}, \mathrm{b}, \mathrm{c}}$

\begin{tabular}{|c|c|c|c|c|}
\hline Compound & $i$ & $n$ & Exptl. & Calc. \\
\hline \multirow[t]{5}{*}{$2 a, M=B$} & ${ }^{10} \mathrm{~B}$ & 1 & 2.3 & 2.8 \\
\hline & ${ }^{11} \mathrm{~B}$ & 1 & 6.9 & 8.4 \\
\hline & ${ }^{14} \mathrm{~N}$ & 4 & 5.5 & 4.6 \\
\hline & ${ }^{10} \mathrm{~B}$ & 2 & 1.9 & 2.2 \\
\hline & ${ }^{11} \mathrm{~B}$ & 2 & 5.6 & 6.6 \\
\hline \multirow[t]{4}{*}{$2 b, M=A l$} & ${ }^{27} \mathrm{Al}$ & 1 & 11.5 & 16.9 \\
\hline & ${ }^{14} \mathrm{~N}$ & 4 & 4.7 & 4.2 \\
\hline & ${ }^{10} \mathrm{~B}$ & 2 & 1.7 & 2.0 \\
\hline & ${ }^{11} \mathrm{~B}$ & 2 & 5.2 & 6.0 \\
\hline \multirow[t]{5}{*}{$\mathbf{2 c}, \mathbf{M}=\mathbf{G a}$} & ${ }^{69} \mathrm{Ga}$ & 1 & 28.0 & 45.6 \\
\hline & ${ }^{71} \mathrm{Ga}$ & 1 & 35.4 & 57.7 \\
\hline & ${ }^{14} \mathrm{~N}$ & 4 & 4.7 & 4.3 \\
\hline & ${ }^{10} \mathrm{~B}$ & 2 & 1.7 & 2.0 \\
\hline & ${ }^{11} \mathrm{~B}$ & 2 & 5.2 & 6.1 \\
\hline \multirow[t]{5}{*}{ 2d, $M=$ In } & ${ }^{113}$ In & 1 & 22.5 & - \\
\hline & ${ }^{115} \mathrm{In}$ & 1 & 41.0 & - \\
\hline & ${ }^{14} \mathrm{~N}$ & 4 & 4.7 & 4.1 \\
\hline & ${ }^{10} \mathrm{~B}$ & 2 & 1.7 & 2.0 \\
\hline & ${ }^{11} \mathrm{~B}$ & 2 & 5.2 & 6.1 \\
\hline
\end{tabular}

${ }^{\mathrm{a}} i=$ isotope; $n=$ number of equivalent spin-active nuclei

${ }^{b}$ experimental values are obtained from least-squares fit optimizations of the spectra ${ }^{c}$ values taken from Ref. [5c,d]

An examination of the experimental (optimized) hfc constants listed in Table 2 reveals that the couplings to the ${ }^{14} \mathrm{~N}$ and ${ }^{11} \mathrm{~B}$ nuclei in the two boraamidinate moieties are similar in magnitude: the difference is less than $0.5 \mathrm{G}$ in all four spectra. This coincidence creates spectral splitting patterns in which most of the expected fine structure becomes disguised under the broad lineshape. For example, the combination of four and two equivalent ${ }^{14} \mathrm{~N}$ and ${ }^{11} \mathrm{~B}$ nuclei, respectively, should theoretically give rise to 63 individual lines. In the case of compounds $\mathbf{2}$, the majority of the signals overlap, creating a splitting pattern which consists only of fifteen broad lines. In the case of the aluminum and gallium spirocycles $\mathbf{2 b}$ and $\mathbf{2 c}$, the hyperfine coupling of the unpaired electron to the 
central metal is also approximately a multiple of the smaller hfes to nitrogen and boron nuclei, which brings about the deceivingly simple splitting patterns in the experimental spectra. However, any simulation attempts using hfcs which are exact multiples of each other are doomed to fail. Although they are able to predict the correct number of individual lines, they do not reproduce the correct lineshapes which are extremely sensitive to even smallest changes in hfcs.

\section{Cubic tetraimidophosphate dianion radicals}

Polyimido anions of the p-block elements have in recent years been of topical interest in main group chemistry [35]. They are related to the more common oxoanions as the imido group $[\mathrm{NR}]^{2-}$ is isoelectronic with the oxo $[\mathrm{O}]^{2-}$ substituent. Second only to silicon, phosphorus forms the largest number of oxoanions, many of which are of significant industrial importance [36]. It is therefore not surprising that numerous imidoanalogues of phosphorus oxoanions have been prepared, including the trisimidometaphosphate $\left[\left(\mathrm{P}(\mathrm{NR})_{3}\right]^{-}[37]\right.$, bisimidophosphinate $\left[\mathrm{R}_{2} \mathrm{P}(\mathrm{NR})_{2}\right]^{2-}[38]$, and the tetraimidophosphate $\left[\mathrm{P}(\mathrm{NR})_{4}\right]^{3-}$ anions [39]. The synthesis of the unsymmetrical tetraimidophosphates $\mathrm{Li}_{3}\left[\mathrm{P}(\mathrm{NR})_{3}\left(\mathrm{NSiMe}_{3}\right)\right]\left(\mathrm{R}={ }^{\mathrm{i}} \mathrm{Pr},{ }^{\mathrm{t}} \mathrm{Bu}, \mathrm{Cy}, \mathrm{Ad}\right)(3)$ has been reported recently [39b]. During the course of this research it was noted that the initially colorless THF solutions of $\mathbf{3}$ became deep blue upon exposure to oxygen. This observation is reminiscent of the behavior reported earlier for the chalcogen-centered polyimido anions in $\left\{\mathrm{Li}_{2}\left[\mathrm{E}\left(\mathrm{N}^{\mathrm{t}} \mathrm{Bu}\right)_{3}\right]\right\}_{2}(\mathrm{E}=\mathrm{S}, \mathrm{Se})$ which form deeply colored persistent radicals upon oxidation [40]. These results provided impetus for a more detailed study of the radical species accessible from controlled reactions of asymmetric tetraimidophosphates with common oxidizing agents.

The one-electron oxidation of $\mathrm{Li}_{3}\left[\mathrm{P}\left(\mathrm{N}^{t} \mathrm{Bu}\right)_{3}\left(\mathrm{NSiMe}_{3}\right)\right]\left(3, \mathrm{R}={ }^{\mathrm{t}} \mathrm{Bu}\right)$ was performed with an half an equivalent of $\mathrm{SO}_{2} \mathrm{Cl}_{2}$, bromine and iodine. This resulted in the successful isolation and EPR spectroscopic characterization of the stable neutral radical complexes $\left\{\left[\mathrm{Me}_{3} \operatorname{SiNP}\left(\mu_{3}-\mathrm{N}^{\mathrm{t} B u}\right)_{3}\right]\left[\mu_{3}-\mathrm{Li}(\mathrm{THF})\right]_{3} \mathrm{X}\right\}^{\bullet}(\mathbf{4 a}, \mathrm{X}=\mathrm{Cl} ; \mathbf{4 b}, \mathrm{X}=\mathrm{Br} ; \mathbf{4 c}, \mathrm{X}=\mathrm{I})[5 \mathrm{~b}, 41]$, of which the iodide salt was the only one analyzed with X-ray crystallography [41]. In the solid state, the complex $\mathbf{4 c}$ was shown to adopt a distorted cubic structure (approx. $C_{3}$ symmetry) in which a tetraimidophosphate dianion radical $\left\{\left[\mathrm{Me}_{3} \operatorname{SiNP}\left(\mathrm{NBu}_{3}\right]^{2}\right\}^{2-\bullet}\right.$ is 
capped by two $\mathrm{Li}^{+}$ions and a molecule of lithium iodide; each of the three $\mathrm{Li}^{+}$cations is also coordinated to one solvent molecule. Recently, it was shown that the dilithiated tetraimidophosphate radical can also trap a monomeric lithium alkoxide, namely $\mathrm{LiO}^{\mathrm{t}} \mathrm{Bu}$, forming a cluster $\left(\mathbf{4 d}, \mathrm{X}=\mathrm{O}^{t} \mathrm{Bu}\right)$ which was found to be isostructural with the iodide derivative in the solid state [42].

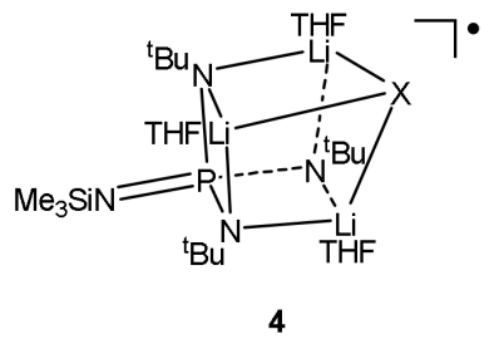

The experimental EPR spectra of THF solutions of $\mathbf{4 a}$ and $\mathbf{4 d}$ at room temperature are shown in Figure 3a [5b,42]. At first glance, it is surprising how little the spectra resemble each other, despite the fact that the two radical systems share similar cubic structures in the solid state. Initially, this was thought to arise from the dissociation of the cubic framework in $\mathbf{4 d}$ to a monocyclic anion radical $\left\{\left[\left(\mathrm{Me}_{3} \mathrm{SiN}\right)\left({ }^{\mathrm{t}} \mathrm{BuN}\right) \mathrm{P}(\mu\right.\right.$ $\left.\left.\left.\mathrm{N}^{\mathrm{t} B u}\right)_{2}\right] \mathrm{Li}(\mathrm{THF})_{2}\right\}^{-\bullet}\left(\right.$ with a $\left[\mathrm{Li}(\mathrm{THF})_{4}\right]^{+}$counterion) and a THF-solvated lithium tertbutoxide [42]. Support for this hypothesis came from studies of the iodide derivative $\mathbf{4 c}$, in which the solvated complex (THF) $)_{3} \mathrm{LiI}$ was isolated and characterized by X-ray crystallography [41]. The THF-solvated $\mathrm{LiO}^{t} \mathrm{Bu}$ salt was, however, not detected in solutions of $\mathbf{4 d}$ [42], raising doubts about the correctness of the dissociation hypothesis. Hence, the systems $\mathbf{4 a}$ and $\mathbf{4 d}$ were subjected to a more thorough study in order to reveal the true identity of these radical species in solution.

Upon closer inspection of the spectra in Figure 3a, it becomes evident that both of them show a distinct doublet feature corresponding to a large $(25 \mathrm{G})$ hfc to ${ }^{31} \mathrm{P}$ nucleus ( $I$ $=1 / 2$ ) [1]. The slowly decaying shape of the signals present in the outer ends of the spectra indicate that hfcs to several magnetically equivalent nuclei contribute to the spectral fine structure [43]. Therefore, the solution structures of the paramagnetic species 4a and 4d should be highly symmetric. Indeed, it may well be that neither of the cubic radicals dissociates to the proposed monocycle upon solvation. It is however extremely difficult to uncover any unambiguous coupling patterns from the spectra as numerous repeating peak differences falling within the range of 1-10 $\mathrm{G}$ can be found. Hence, 
theoretical calculations for radicals 4 using isostructural solid-state geometries were carried out in order to facilitate spectral simulation and either confirm or refute the hypothesis that both $\mathbf{4 a}$ and $\mathbf{4 d}$ share similar cubic structures also in solution [5b].

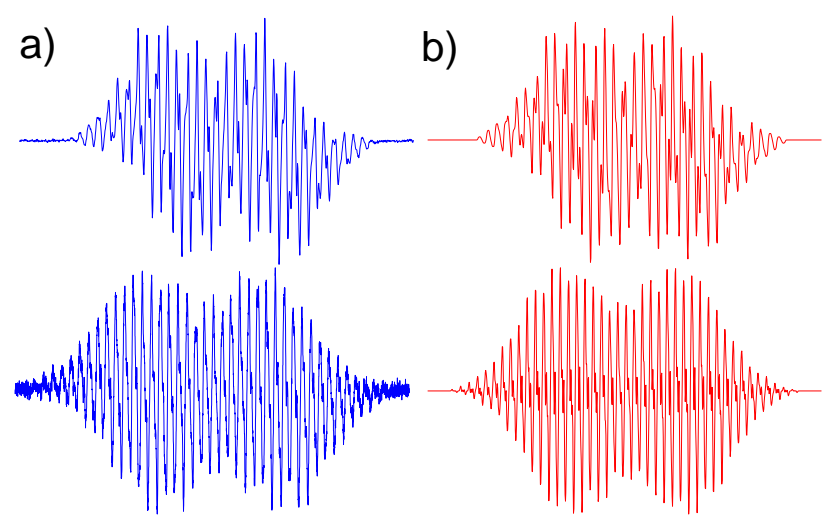

Fig. 3. Experimental (a) and simulated (b) EPR spectra of radicals 4a (top) and 4d (bottom) [5b].

DFT calculations indicated that the spin density in $\mathbf{4}$ is primarily located on three symmetric p-orbital-type lobes around the nitrogen nuclei within the cubic framework [5b]. Consequently, the EPR spectra of these radicals are expected to exhibit equal hyperfine couplings to the three equivalent ${ }^{14} \mathrm{~N}$ atoms. Though the calculations indicate only minimal spin density (via spin polarization) on the phosphorus and lithium atoms, significant ${ }^{31} \mathrm{P}$ and ${ }^{7} \mathrm{Li}$ hfcs are anticipated due to the large gyromagnetic ratios of these nuclei [1]. Conversely, the hfcs to the lone trimethylsilyl nitrogen atom and the remote halogen atoms are calculated to be very small (less than $0.5 \mathrm{G}$ ) and should, thus, have only a small contribution to the width of experimental EPR spectra.

The calculated hfcs for compounds $\mathbf{4 a}$ and $\mathbf{4 d}$ are listed in Table 2 along with the values obtained with iterative least-squares fit methods [5b]; there is an excellent agreement between the two set of numbers. Figure $3 b$ shows that simulations created by using the least-squares optimized hfc constants are able to reproduce even the minutest features present in the experimental spectra. It is therefore safe to conclude that the spectral and computational analyses are able to prove the identity of these paramagnetic species in solution conclusively. The retention of cubic geometry in solution for $\mathbf{4 a}$ and 
4d is also consistent with the fact that the expected by products of solvation, $\mathrm{LiCl}$ or $\mathrm{LiO}^{t} \mathrm{Bu}$, could not be isolated from the paramagnetic solutions.

Table 2

Experimental and calculated hyperfine coupling constants (in Gauss) of radicals $\mathbf{4 a}$ and $\mathbf{4 d}^{\mathrm{a}, \mathrm{b}, \mathrm{c}}$

\begin{tabular}{|c|c|c|c|c|}
\hline Compound & $i$ & $n$ & Exptl. & Calc. \\
\hline \multirow[t]{6}{*}{$4 a, X=C l$} & ${ }^{31} \mathrm{P}$ & 1 & 24.7 & 28.6 \\
\hline & ${ }^{14} \mathrm{~N}$ & 3 & 5.3 & 3.2 \\
\hline & ${ }^{14} \mathrm{~N}$ & 1 & 0.4 & 0.6 \\
\hline & ${ }^{7} \mathrm{Li}$ & 3 & 2.2 & 2.5 \\
\hline & ${ }^{35} \mathrm{Cl}$ & 1 & 0.2 & 0.2 \\
\hline & ${ }^{37} \mathrm{Cl}$ & 1 & 0.1 & 0.2 \\
\hline \multirow[t]{4}{*}{$4 \mathrm{~d}, X=\mathrm{O}^{t} \mathrm{Bu}$} & ${ }^{31} \mathrm{P}$ & 1 & 25.2 & 29.3 \\
\hline & ${ }^{14} \mathrm{~N}$ & 3 & 5.4 & 3.1 \\
\hline & ${ }^{14} \mathrm{~N}$ & 1 & 0.3 & 0.6 \\
\hline & ${ }^{7} \mathrm{Li}$ & 3 & 1.9 & 2.3 \\
\hline
\end{tabular}

a $i=$ isotope; $n=$ number of equivalent spin-active nuclei

$\mathrm{b}$ experimental values are obtained from least-squares fit optimizations of the spectra

${ }^{c}$ values taken from Ref. [5b]

The hfc pattern in the EPR spectra of $\mathbf{4 a}$ and $\mathbf{4 d}$ is primarily a doublet due to the large dominant coupling of the unpaired electron to the central ${ }^{31} \mathrm{P}$ nucleus. The signal is further split into septets by the three equivalent ${ }^{14} \mathrm{~N}$ nuclei and the (mostly hidden) decet structure arises from the smaller hfcs to the three ${ }^{7} \mathrm{Li}$ centers. The numerical values listed in Table 2 illustrate the sensitivity of the spectral appearance to very small changes in the parameters: the different fine structure in the two spectra arises entirely from a variation of less than $0.3 \mathrm{G}$ in both the spectral linewidth and the hfcs to the three equivalent ${ }^{7} \mathrm{Li}$ nuclei as well as to the remote ${ }^{14} \mathrm{~N}$ and ${ }^{35,37} \mathrm{Cl}$ centers.

In contrast to the results obtained for $\mathbf{4 a}$ and $\mathbf{4 d}$, it proved impossible to simulate the EPR spectra of the corresponding bromide and iodide derivatives accurately by assuming retention of the cubic framework [5b,41]. This is attributed to the fact that the EPR spectra recorded for $\mathbf{4 b}$ and $\mathbf{4 c}$ do not display the expected inversion symmetry characteristic of a single stable paramagnetic species in solution. It is however possible to identify splitting patterns arising from coupling of the unpaired electron with one 
phosphorus and three equivalent nitrogen nuclei in both spectra. Thus, it is believed that the major components present in the THF solutions of $\mathbf{4 b}$ and $\mathbf{4} \mathbf{c}$ are the expected cubic radicals $[5 \mathrm{~b}]$. Due to the weaker nature of $\mathrm{Li}-\mathrm{X}$ interactions when $\mathrm{X}=\mathrm{Br}$, I as compared to $\mathrm{X}=\mathrm{Cl}, \mathrm{O}^{\mathrm{t}} \mathrm{Bu}$, the cubic radicals $\mathbf{4 b}$ and $\mathbf{4 c}$ dissociate over time and form currently unknown radical species which are present as minor components in the experimental spectra. The precipitation of crystalline $(\mathrm{THF})_{3} \mathrm{LiI}$ from the THF solutions of the iodide derivative supports this statement and implies that the initial dissociation product might in both cases be a dilithiated radical species, i.e. $\left\{\left[\mathrm{Li}(\mathrm{THF})_{2}\right]_{2}\left[\mathrm{P}\left(\mathrm{NSiMe}_{3}\right)\left(\mathrm{N}^{\mathrm{t} B u}\right)_{3}\right]\right\}^{\bullet}$, for which a spirocyclic structure has been proposed $[5 b, 41]$.

\section{Paramagnetic complexes of gallium with diazabutadiene ligands}

The 1,4-diaza-1,3-butadiene (DAB) ligands (5) are useful reagents in organometallic chemistry as the lone pairs on the two nitrogen atoms and the four $\pi$ electrons of the $\mathrm{C}=\mathrm{N}$ double bonds allow these molecules to coordinate to metal centers using 2, 4, 6 or 8 electrons. The DAB ligands can coordinate to metal centers as anions and dianions by accepting either one or two electrons from the metal, respectively. Of especial interest are the complexes in which DAB ligands are coordinated to the metal center as monoanions (6) since the resulting species commonly have non-singlet ground states. Paramagnetic DAB complexes of alkaline earth metals [44,45], lithium [45], and zinc $[44 b, 45,46]$ have been known for a number of years, while more later work in this field has resulted in the isolation of a plethora of Group 13 complexes containing the DAB anion radical [47].

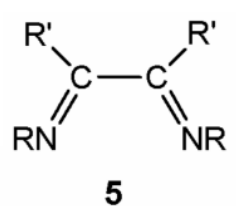

5

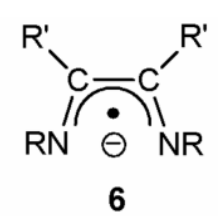

6

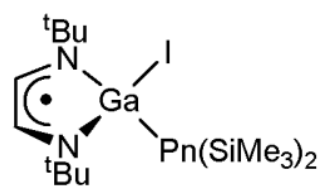

7

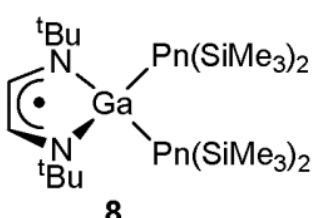

8

Recently, the isolation and structural characterization of the monocyclic paramagnetic gallium complexes $\left\{\left({ }^{\mathrm{t}} \mathrm{Bu}-\mathrm{DAB}\right) \mathrm{Ga}[\mathrm{I}]\left[\mathrm{Pn}\left(\mathrm{SiMe}_{3}\right)_{2}\right]\right\}^{\bullet}(\mathbf{7 a}, \mathrm{Pn}=\mathrm{N} ; \mathbf{7 b}, \mathrm{Pn}=$ $\mathrm{P} ; \mathbf{7 c}, \mathrm{Pn}=\mathrm{As})$ and the related dipnictogen species $\left\{\left({ }^{\mathrm{t}} \mathrm{Bu}-\mathrm{DAB}\right) \mathrm{Ga}\left[\mathrm{Pn}\left(\mathrm{SiMe}_{3}\right)_{2}\right]_{2}\right\}^{\bullet}(\mathbf{8 b}$, $\mathrm{Pn}=\mathrm{P} ; \mathbf{8 c}, \mathrm{Pn}=\mathrm{As})$ were reported $[47 \mathrm{~g}]$. The EPR spectra of these novel radicals, see Figures $4 \mathrm{a}$ and $5 \mathrm{a}$, proved to be extremely complex which prevented the authors from 
obtaining accurate simulations using simple trial-and-error methods [47g]. In light of this fact, a computational study of the complexes $\mathbf{7}$ and $\mathbf{8}$ was undertaken in order to determine their electronic structures, spin densities, and hfcs, which together would give a realistic picture of spin distribution in these systems [5e]. The complex EPR spectra reported for $\mathbf{7}$ and $\mathbf{8}$ were then interpreted in terms of the computationally predicted hfcs.

The hfc constants of the model radical systems $\left\{\left({ }^{\mathrm{t}} \mathrm{Bu}-\mathrm{DAB}\right) \mathrm{Ga}[\mathrm{I}]\left[\mathrm{Pn}\left(\mathrm{SiH}_{3}\right)_{2}\right]\right\}^{\bullet}(\mathrm{Pn}$ $=\mathrm{N}, \mathrm{P}, \mathrm{As})$ and $\left\{\left({ }^{\mathrm{t}} \mathrm{Bu}-\mathrm{DAB}\right) \mathrm{Ga}\left[\mathrm{Pn}\left(\mathrm{SiH}_{3}\right)_{2}\right]_{2}\right\}^{\circ}(\mathrm{Pn}=\mathrm{P}, \mathrm{As})$ were calculated by using both relativistic (radicals 7) and non-relativistic (radicals 8) methods; the results from theoretical calculations are summarized in Table $3[5 \mathrm{e}]$. The calculated values of both ${ }^{1} \mathrm{H}$ and ${ }^{14} \mathrm{~N}$ hfcs show only minor variation between the different systems, as expected, since both $\mathbf{7}$ and $\mathbf{8}$ are ligand-centered radicals; the numerical values of the couplings are also consistent with the hfcs observed in other related DAB-centered radical systems [47]. In radicals $\mathbf{8}$, the dominating ${ }^{69,71} \mathrm{Ga}$ couplings $(25-30 \mathrm{G})$ arise from a combination of spin polarization effects and relatively high gyromagnetic ratios of the two isotopes of gallium [1]. Conversely, only small hfcs are calculated to the pnictogen atoms both in $\mathbf{7}$ and $\mathbf{8}$. These couplings vary roughly with the relative magnitudes of the gyromagnetic ratios of ${ }^{14} \mathrm{~N},{ }^{31} \mathrm{P}$, and ${ }^{75}$ As nuclei [1], which implies a relatively constant spin density on the remote pnictogen centres. In comparison to radicals $\mathbf{8}$, significantly smaller hfcs to the gallium metal are calculated for systems 7. Instead, considerable hyperfine interactions to the ${ }^{127}$ I nuclei in 7 are calculated which should also be visible in the experimental spectra.

Excellent simulations of the experimental EPR spectra recorded for mono(Figure 4b) and dipnictogen (Figure 5b) complexes were obtained using the calculated hfc constants as initial estimates of the true couplings, and optimizing the sets of values using iterative methods [5e]. The slight differences between the left- and right-hand sides of the experimental spectra of compounds 7 (i.e. the lack of perfect inversion symmetry with respect to signal intensities) is believed to be caused by higher-order hyperfine interactions due to the presence of large hfcs to the heavy nucleus ${ }^{127} \mathrm{I}$ [48]. 


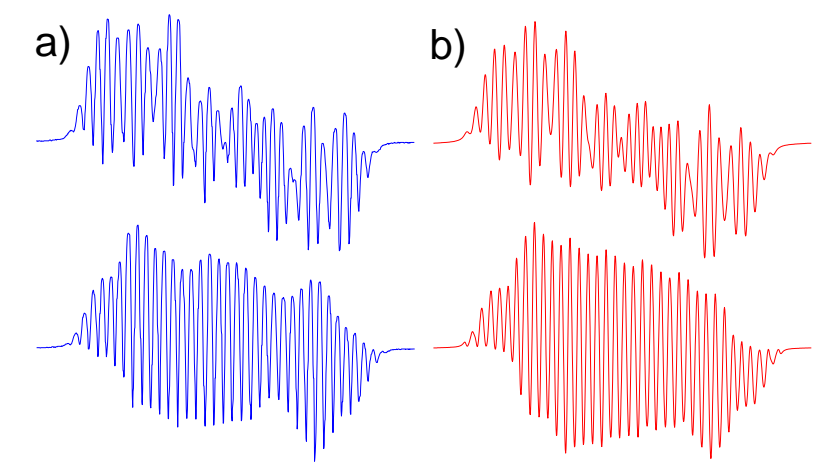

Fig. 4. Experimental (a) and simulated (b) EPR spectra of radicals 7a (top) and 7b (bottom) $[5 \mathrm{e}, 47 \mathrm{~g}]$.

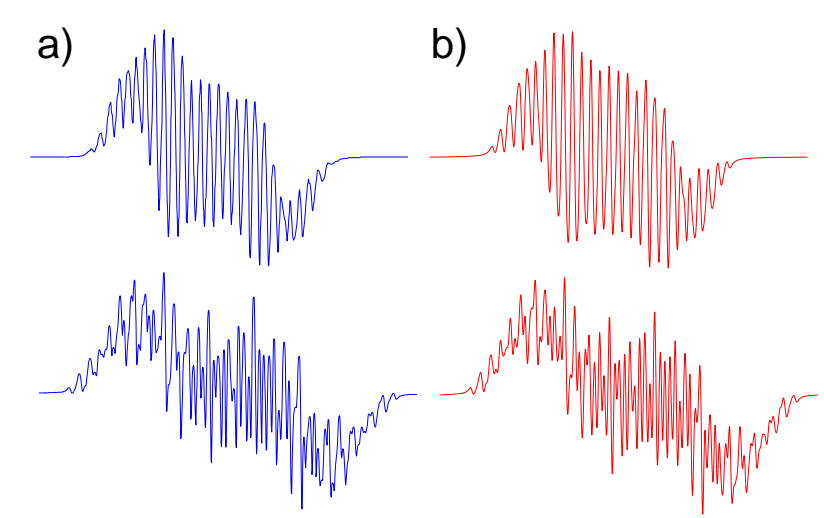

Fig. 5. Experimental (a) and simulated (c) EPR spectra of radicals 8b (top) and 8c (bottom) $[5 \mathrm{e}, 47 \mathrm{~g}]$.

As expected (see above), the experimental EPR spectrum of 7a does not display a quartet pattern characteristic of a large coupling to gallium (see Figure 4). Instead, the spectrum shows a partially resolved sextet pattern due to hfc to one ${ }^{127}$ I nucleus $(I=5 / 2)$ which dominates the spectrum [1]; the sextet pattern, although present, is hidden in the spectrum of $\mathbf{7 b}$ by the significantly larger hfc to the remote pnictogen nucleus $(7 \mathrm{G}$ for ${ }^{31} \mathrm{P}$ vs. $1 \mathrm{G}$ for ${ }^{14} \mathrm{~N}$ ). In the experimental EPR spectra of radicals $\mathbf{8 b}$ and $\mathbf{8 c}$ (see Figure 5), the quartet pattern due to the hfc to the gallium nucleus is only visible in the spectrum recorded for the arsenic species: in case of $\mathbf{8 c}$, the hyperfine coupling to the two remote pnictogen nuclei is not a multiple of the hfcs to the ${ }^{1} \mathrm{H}$ and ${ }^{14} \mathrm{~N}$ nuclei in the DAB backbone which leads to better resolved splitting patterns ( $c f$. data for $\mathbf{8 b}$ ). 
Table 3 Experimental and calculated hyperfine coupling constants (in Gauss) of radicals 7 and $\mathbf{8}^{\text {a, b, c }}$

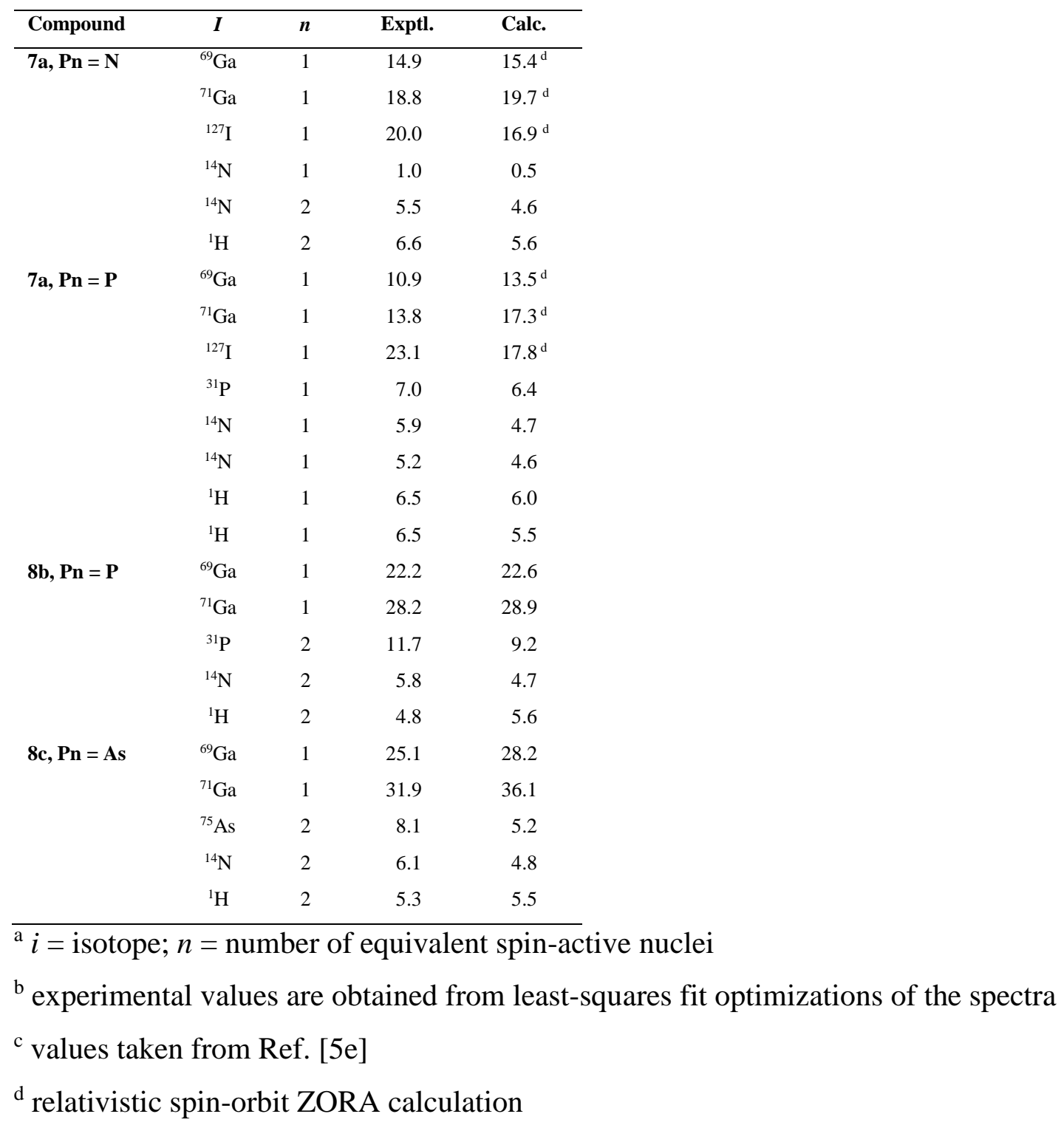

As shown above, the complexity in the EPR spectra of $\mathbf{7}$ and $\mathbf{8}$ does not arise from different isotropic $g$-values for the ${ }^{69} \mathrm{Ga}$ and ${ }^{71} \mathrm{Ga}$ isotopomers as previously suggested $[47 \mathrm{~g}]$, but from the presence of higher-order splitting effects and hfcs which are approximately multiples of each other [5e]. Taken in concert with some previous experimental work [47], the spectral analyses described herein suggested that the published experimental EPR results of some related paramagnetic Group 13-DAB 
complexes might also have been misinterpreted [47h]. Detailed theoretical investigations of these systems have recently been reported [49].

\section{Conclusions}

Theoretical calculations have been used in combination with mathematical methods to aid in the analysis of some very complex experimental EPR spectra of main group radicals. The results and methodologies reviewed in this paper clearly demonstrate the capability of density functional methods to predict hyperfine coupling constants that are in semi-quantitative agreement with the experimental values even for complex multinuclei compounds including heavy main group elements. Thus, calculated coupling constants provide essential initial estimates of the true hyperfine couplings present in main group radical systems. Their subsequent use in least-squares fit based spectral simulation methods yields an accurate description of the magnetic hyperfine interactions, provided that the experimental data contains enough characteristic information to allow unambiguous convergence of the iterative methods and that the spectrum truly corresponds to the purported radical species. Through this combination of experimental and computational methodologies, the research described in this account has provided fundamental information about many new stable and persistent main group radicals that is not accessible by other methods. We hope that the examples reviewed here illustrate the enormous potential of this methodology and that the high quality of the reported results will encourage other chemists working in the field of EPR spectroscopy to make use of this approach.

\section{Acknowledgement}

Financial support from NSERC (Canada), the Alberta Ingenuity Fund, the Academy of Finland, and the Universities in Calgary, Lethbridge and Jyväskylä is gratefully acknowledged. 


\section{References and notes}

[1] For properties of different nuclei, see J. Weil, J. R. Bolton, J. E. Wertz, Electron Paramagnetic Resonance: Elemental Theory and Practical Applications, WileyInterscience, New York, 1994, (Appendix G).

[2] For radicals in which the spin density is highly delocalized over several hydrogen and nitrogen nuclei, the EPR spectra can, however, become increasingly complex. See, for example, the EPR spectrum reported for the cation radical of 6-hydrodipyrido[1,2-c:2',1'e]-imidazole in P.D. Sullivan, J.Y. Fong, M.L. Williams, J. Phys. Chem. 82 (1978) 1181. [3] For some recent examples, see (a) B. Tumanskii, P. Pine, Y. Apeloig, N. J. Hill, R. West, J. Am. Chem. Soc. 126 (2004) 7786; (b) T. K. Paine, T. Weyhermuller, L. D. Slep, F. Neese, E. Bill, E. Bothe, K. Wieghardt, P. Chaudhuri, Inorg. Chem. 43 (2004) 7324; (c) B. Tumanskii, P. Pine, Y. Apeloig, N. J. Hill, R. West, J. Am. Chem. Soc. 127 (2005) 8248; (d) L. Beer, R. C. Haddon, M. E. Itkis, A. A. Leitch, R. T. Oakley, R. W. Reed, J. F. Richardson, D. G. VanderVeer. Chem. Commun. (2005) 1218; (e) P. Maire, M. Königsmann, A. Sreekanth, J. Harmer, A. Schweiger, H. Grützmacher, J. Am. Chem. Soc. 128 (2006) 6578.

[4] See, for example, discussion in Ref. [1].

[5] (a) T. Chivers, D. J. Eisler, C. Fedorchuck, G. Schatte, H. M. Tuononen, R. T. Boeré, Chem. Commun (2005) 3930; (b) A. F. Armstrong, T. Chivers, H. M. Tuononen, M. Parvez, R. T. Boeré, Inorg. Chem. 44 (2005) 7981; (c) A. F. Armstrong, T. Chivers, H. M. Tuononen, M. Parvez, Inorg. Chem. 44 (2005) 5778; (d) T. Chivers, D. J. Eisler, C. Fedorchuk, G. Schatte, H. M. Tuononen, R. T. Boeré, Inorg. Chem. 45 (2006) 2119; (e) H. M. Tuononen, A. F. Armstrong, Inorg. Chem. 44 (2005) 8277.

[6] For a more in-depth discussion of the theory behind EPR spectroscopy, see Ref. [1].

[7] M. Kaupp, M. Bühl, V. G. Malkin (Eds.), Calculation of NMR and EPR Parameters: Theory and Applications, Wiley-VCH, Weinheim, 2004 (Chapter 31).

[8] For this reason, Slater-type orbitals (STO) should, in principle, show superior performance in calculation of hfc constants. Comparison of results at various DFT levels of theory has, however, shown that in practice both STO- and GTO-based approaches yield results of comparable accuracy. See Chapter 29 in Ref. [7].

[9] W. Meyer, J. Chem. Phys. 51 (1969) 5149. 
[10] For recent benchmarks, see (a) V. Barone, in: D. P. Chong (Ed.), Recent Advances in Density Functional Methods, Vol. 1, World Scientific, Singapore, 1996 (Chapter 8); (b) L. Hermosilla, P. Calle, J. M. Garcia de la Vega, C. Sieiro, J. Phys. Chem. A 109 (2005) 1114; (c) L. Hermosilla, P. Calle, J. M. Garcia de la Vega, C. Sieiro, J. Phys. Chem. A 109 (2005) 7626.

[11] M. T. Nguyen, S. Creve, L. G. Vanquickenborne, J. Phys. Chem. A 101 (1997) 3174.

[12] T. Helgaker, M. Jaszuński, K. Ruud, A. Górska, Theor. Chem. Acc. 99 (1998) 175.

[13] For a recent review on main group radicals, see P. P. Power, Chem. Rev. 103 (2003) 789.

[14] See discussion in Chapters 5 and 29 of Ref. [7].

[15] See discussion in Chapter 30 of Ref. [7].

[16] See discussion in Chapter 29 of Ref. [7].

[17] A. V. Arbuznikov, M. Kaupp, V. G. Malkin, R. Reviakine, O. L. Malkina, Phys. Chem. Chem. Phys. 4 (2002) 5467.

[18] See discussion in Chapters 29 and 35 of Ref. [7].

[19] An important exception to this rule is observed with transition metal compounds for which none of the current density functionals shows uniform performance.

[20] The analysis of EPR spectra using iterative least-squares fit methods has been reviewed in B. Kriste, Anal. Chim. Acta 265 (1992) 191.

[21] For radicals containing nuclei that have more than one naturally abundant spinactive isotope, the simulation is done by using a weighted sum of individual subspectra for each isotopomer. The weight factors can be readily calculated from the relative abundances of the different isotopes.

[22] In this paper we have omitted lineshape related questions from the discussion mainly for simplicity. In addition, all EPR spectra presented in Section 3 can be simulated using either pure or almost pure (i.e. small Gaussian component) Lorenzian lineshape. However, we wish to point out here that in many cases the experimental EPR spectra can show linewidth variations due to internal dynamics (alternating lineshape) or slow molecular tumbling (asymmetric lineshape). In such cases, accurate simulations of the EPR spectra require the use of approaches which are capable of dealing with more complex lineshape functions. 
[23] (a) A. Nelder, R. Mead, Comput. J. 7 (1965) 308; (b) B. Kirste, J. Magn. Reson. 73 (1987) 213; (c) K. Levenberg, Quart. Appl. Math. 2 (1944) 164; (d) D. Marquardt, SIAM J. Appl. Math. 11 (1963) 1; (d) R. Hooke, T. A. Jeeves, J. Assoc. Comput. Mach. 8 (1961) 212; (e) P. E. Gill, W. Murray, SIAM J. Numer. Anal. 15 (1978) 977.

[24] (a) G. R. Hanson, K.E. Gates, C. J. Noble, M. Griffin, A. Mitchell, S. Benson, J. Inorg. Biochem 85 (2004) 903; (b) S. Stoll, A. Schweiger, J. Magn. Reson. 178 (2006) 42; (c) D. R. Duling, J. Magn. Reson. 104 (1994) 105.

[25] A. L. J. Beckwih, S. Brumby, J. Magn. Reson. 73 (1987) 252.

[26] We note here that application of various peak difference search methods yields only ambiguous estimates of hfcs, because a multitude of sums and differences are generally found from the experimental spectrum in addition to the relevant parameters. In addition, whereas ENDOR spectroscopy can effectively provide high-quality values for hfcs, the number of each equivalent nuclei is not by any means clear from the measured spectra. Hence, this information must be determined separately either by using chemical considerations or time consuming "brute force" approaches in which several different EPR simulations with varying number of nuclei are performed.

[27] J. Eloranta, University of Jyväskylä, Finland, XEMR v. 0.7, 2004.

[28] Bruker Analytische Messtechnik GmbH, WINEPR SimFonia v. 1.25, 1996.

[29] (a) J. P. Perdew, K. Burke, M. Ernzerhof, Phys. Rev. Lett. 77 (1996) 3865; (b) J. P. Perdew, K. Burke, M. Ernzerhof, Phys. Rev. Lett. 78 (1997) 1396; (c) J. P. Perdew, K. Burke, M. Ernzerhof, J. Chem. Phys. 105 (1996) 9982; (d) M. Ernzerhof, G. E. Scuseria, J. Chem. Phys. 110 (1999) 5029.

[30] Gaussian 03, Revision C.02, M. J. Frisch, et al. Gaussian, Inc., Wallingford CT, 2004.

[31] ADF2004.01, SCM, Theoretical Chemistry, Vrije Universiteit, Amsterdam, 2004.

[32] T. Chivers, C. Fedorchuk, G. Schatte, M. Parvez, Inorg. Chem. 42 (2003) 2084.

[33] Model systems in which the computationally more demanding t $\mathrm{Bu}$ groups are replaced with Me groups were used to speed up the calculations. In the present case, such alteration is expected to have a negligible effect on the electronic structures and the spin density distributions of the radical species 2a-d. 
[34] We note that the indium species $\mathbf{2 d}$ is a transient radical and even though its characterization was carried out at $-40^{\circ} \mathrm{C}$, the recorded EPR spectrum still shows some signals belonging to the decomposition products.

[35] For recent reviews, see (a) J. K. Brask, T. Chivers, Angew. Chem., Int. Ed. 40 (2001) 3960; (b) G. M. Aspinall, M. C. Copsey, A. P. Leedham, C. A. Russell, Coord. Chem. Rev. 227 (2002) 217.

[36] For a review, see T. Chivers, Top. Curr. Chem. 229 (2003) 143.

[37] E. Niecke, M. Frost, M. Nieger, V. von der Gönna, A. Ruban, W. W. Schoeller, Angew. Chem., Int. Ed. Engl. 23 (1994) 2111.

[38] A. Steiner, S. Zacchini, P. I. Richards, Coord. Chem. Rev. 227 (2002) 193.

[39] (a) P. R. Raithby, C. A. Russell, A. Steiner, D. S. Wright, Angew. Chem., Int. Ed.

Engl. 36 (1997) 649; (b) A. Armstrong, T. Chivers, M. Krahn, M. Parvez, G. Schatte, Chem. Commun. (2002) 2332.

[40] (a) R. Fleischer, S. Freitag, F. Pauer, D. Stalke, Angew. Chem., Int. Ed. Engl. 35 (1996) 204; (b) R. Fleischer, S. Freitag, D. Stalke, J. Chem. Soc., Dalton Trans. (1998) 193; (c) T. Chivers, M. Parvez, G. Schatte, Inorg. Chem. 35 (1996) 4094; (d) J. K. Brask, T. Chivers, B. McGarvey, G. Schatte, R. Sung, R. T. Boeré, Inorg. Chem. 37 (1998) 4633.

[41] A. Armstrong, T. Chivers, M. Parvez, R. T. Boeré, Angew. Chem., Int. Ed. 42 (2004) 502.

[42] A. Armstrong, T. Chivers, M. Parvez, G. Schatte, R. T. Boeré, Inorg. Chem. 43 (2004) 3459.

[43] Another possibility is that the hfcs are almost equal by chance. In such a case one would, however, expect that the spectral lines would be much broader ( $c f$. EPR spectra in Figure 2).

[44] (a) C. Corvaja, L. Pasimeni, Chem. Phys. Lett. 39 (1976) 261; (b) M. Kaupp, H. Stoll, H. Preuss, W. Kaim, T. Stahl, G. van Koten, E. Wissing, W. J. J. Smeets, A. L. Spek, J. Am. Chem. Soc. 113 (1991) 5606.

[45] M. G. Gardiner, G. R. Hanson, M. J. Henderson, F. C. Lee, C. L. Raston, Inorg. Chem. 33 (1994) 2456.

[46] S. Richter, C. Daul, A. v. Zelewsky, Inorg. Chem. 15 (1976) 943. 
[47] (a) N. F. G. Cloke, G. R. Hanson, M. J. Henderson, P. B. Hitchcock, C. L. Raston, J. Chem. Soc., Chem. Commun. (1989) 1002; (b) N. F. G. Cloke, C. I. Dalby, M. J. Henderson, P. B. Hitchcock, C. H. L. Kennard, R. N. Lamb, C. L. Raston, J. Chem. Soc., Chem. Commun. (1990) 1394; (c) M. G. Gardiner, C. L. Raston, B. W. Skelton, A. H. White, Inorg. Chem. 36 (1997) 2795; (d) T. Pott, P. Jutzi, W. Kaim, W. W. Schoeller, B. Neumann, A. Stammler, H.-G. Stammler, M. Wanner, Organometallics 21 (2002) 3169; (e) R. J. Baker, R. D. Farley, C. Jones, M. Kloth, D. M. Murphy, J. Chem. Soc., Dalton. Trans. (2002) 3844; (f) R. J. Baker, R. D. Farley, C. Jones, M. Kloth, D. M. Murphy, Chem. Commun. (2002) 1196; (g) K. L. Antcliff, R. J. Baker, C. Jones, D. M. Murphy, R. P. Rose, Inorg. Chem. 44 (2005) 2098; (h) R. J. Baker, R. D. Farley, C. Jones, D. P. Mills, M. Kloth, D. M. Murphy, Chem. Eur. J. 11 (2005) 2972.

[48] To accurately model the experimental spectra, a third-order perturbation theory based Hamiltonian was utilized when creating the spectral simulations.

[49] H. M. Tuononen, A. F. Armstrong, Dalton Trans. (2006) 1885. 